\title{
Auf der Suche nach
}

kooperativen Konzepten

\section{Die Ganztagsschule wird die Jugendhilfe nachhaltig verändern}

\author{
WERNER MIEHLE-FREGIN \\ Der Diplompädagoge Werner \\ Miehle-Fregin ist wissenschaftlicher \\ Mitarbeiter für Jugendhilfeplanung, \\ Jugendhilfeberichterstattung und \\ Forschung im Landesjugendamt im \\ Kommunalverband für Jugend und \\ Soziales Baden-Württemberg.
}

\author{
Die Auswirkungen des Ausbaus der Ganztagsschulen \\ auf die Strukturen und Arbeitsweisen der Kinder- und \\ Jugendhilfe wurden in einem Forschungsprojekt in Baden- \\ Württemberg untersucht. Dabei zeigten sich für deren \\ wichtigste Arbeitsfelder interessante Unterschiede.
}

Nachdem das Investitionsprogramm des Bundes »Zukunft Bildung und Betreuung « in Baden-Württemberg auf starken Zuspruch gestoßen war, legte das Land 2006 selbst ein Programm »Bedarfsorientierter Ausbau und Weiterentwicklung von Ganztagsschulen« auf.

Seitdem schreitet der Ausbau der Ganztagesschulen in Baden-Württemberg rasch voran, die konzeptionelle und rechtliche Ausgestaltung befindet sich aber noch in einem Klärungsprozess. Die grün-rote Landesregierung hat sich nun zum Ziel gesetzt, die Ganztagsschule als Regelform im Schulgesetz zu verankern: "Ziel ist eine rhythmisierte Ganztagsschule ohne Hausaufgaben, in der Unterricht, Lernzeit, Arbeitsgemeinschaften sowie Freizeit- und Bildungsangebote sinnvoll über den Schultag verteilt sind. Dabei sollen die Ganztagsschulen vielfältige Kooperationen eingehen können.«(1)

Wenn die Schülerinnen und Schüler tagsüber länger an der Schule sind, gewinnt neben der Wissensvermittlung auch der Erziehungsauftrag der Schule stärker an Bedeutung und die Schule wird zum Betreuungs- und Lebensort junger Menschen. Erziehung, Betreuung und Persönlichkeitsbildung junger Menschen zählen zu den klassischen Aufgaben der Jugendhilfe. Es stellt sich also die Frage, welche Kooperations- und Innovationschancen, aber auch welche Konkurrenzen sich für die Jugendhilfe im Hinblick auf die Ganztagsschulen ergeben.
Dieser Frage ging der Kommunalverband für Jugend und Soziales BadenWürttemberg in einem Forschungsvorhaben nach, das er zusammen mit der Hochschule Osnabrück (Projektleitung Prof. Dr. Stephan Maykus) und dem Institut für Soziale Arbeit e. V. in Münster durchführte. Das Forschungsvorhaben sollte klären, welche Auswirkungen der Ausbau der Ganztagsschulen auf die Kinder- und Jugendhilfe in BadenWürttemberg hat und wie die Kinderund Jugendhilfe in diesem Prozess selbst eine aktive und (mit-) steuernde Rolle einnehmen kann.

Der Ausbau der Ganztagsschulen ist bislang nicht so weit fortgeschritten, dass die Auswirkungen auf die Kinder- und Jugendhilfe bereits mit harten Daten zu Veränderungen beim Personal und bei den unterschiedlichen Jugendhilfeleistungen abgebildet werden könnten. Die empirischen Grundlagen des Forschungsvorhabens wurden in Befragungen von Jugendämtern und ergänzenden Befragungen freier Träger ermittelt. Dabei zeigten sich für die wesentlichen Arbeitsfelder der Kinder und Jugendhilfe teilweise interessante Unterscheidungen.

\section{Auswirkungen bei der Jugendhilfeplanung}

Bislang ist die Ganztagsschule kein Schwerpunktthema der Jugendhilfeplanung, auch sind kaum abgestimmte Schulentwicklungs- und Jugendhilfeplanungen vorhanden. Eine sinnvolle Ver- 
zahnung, die vor Ort individuell entwickelt werden muss, ist jedoch eine wichtige Voraussetzung für eine wirksame Kooperation von Jugendhilfe und Schule.

Die Forscher (2) empfehlen die Bestimmung des Verhältnisses von Jugendhilfe- und Bildungsplanung in den Stadt- und Landkreisen mit dem Ziel der Aufgabenschärfung einer schulbezogenen Jugendhilfeplanung. Ferner ein erweitertes Datenkonzept, mit dem Jugendhilfeplanung Teil integrierter Planungsstrukturen werden kann.

Jugendhilfeplanung soll eine Auseinandersetzung in der Fachöffentlichkeit initiieren: Was ist unser Bild von Kinderund Jugendhilfe der Zukunft? Wie sehen demnach kooperative Konzepte und Leistungen mit (Ganztags-) Schulen aus?

Integrierte und kontinuierliche Planungsprozesse zu Bildung und sozialer Teilhabe sind im jeweiligen Stadt- und Landkreis nur mit erhöhten Personalressourcen und Planungsstrukturen in den Verwaltungen möglich. Darauf weisen die Forscher ausdrücklich hin.

\section{Auswirkungen bei der Kinder- und Jugendarbeit}

Die Jugendreferate der Stadtkreise und der Landkreise sind in unterschiedlicher Dichte mit Entwicklungen im Hinblick auf den Ausbau der Ganztagsschule befasst. Während Ganztagsschule für die Kreisjugendreferenten kein prioritäres Thema ist, sehen die kommunalen Jugendreferenten in den Städten eindeutig eine zunehmende Bedeutung der Kooperation von Jugendarbeit und Ganztagsschule und sich selbst dabei in einer planenden und koordinierenden Funktion.

Konzepte offener Jugendarbeit verändern sich zu Angeboten über Mittag, aber auch »ein wachsender Anspruch auf verlässliche Ferienbetreuung « wird genannt. Insgesamt steht eine Neujustierung der Konzepte offener Jugendarbeit zwischen Sozialraum und Schule an.

Die Spielräume für das Engagement der Jugendverbände in Ganztagsschulen scheinen - insbesondere wegen ihres ehrenamtlichen Charakters - eng zu sein. Gleichzeitig sehen sich die Jugendverbände mit dem Druck konfrontiert, die Bezüge zu Ganztagsschulen zu intensivieren. Dadurch erreichen sie zwar mehr Kinder und Jugendliche, diese können sie aber nicht in das eigentliche Verbandsleben außerhalb der Schule einbinden. Dieses "Mitgliedschaftsund Rekrutierungsparadox" der Jugendverbände wird ein Schlüsselthema der zukünftigen Entwicklung der Jugendarbeitslandschaft sein.

Allgemein werden in der Kinder- und Jugendarbeit auch Bedenken deutlich, das Selbstbild durch die Arbeit in der Ganztagsschule nicht vollständig beibehalten zu können und den Charakter der Freiwilligkeit und der Offenheit zu verlieren. Um Kinder- und Jugendarbeit im Kontext des Ganztagsschulausbaus adäquat gestalten zu können, müssen schulbezogene Angebote besser erfasst und gezielt in Konzeptentwicklungsprozesse einbezogen werden.

Die Kinder- und Jugendarbeit steht vor der Aufgabe, ihr Profil unter Einbeziehung schulbezogener Angebote zu schärfen. Sie darf jedoch in der Kooperation mit Schulen ihren eigenständigen Auftrag nicht vernachlässigen oder zweckentfremdend eingesetzt werden. Dazu braucht es Kooperationsvereinbarungen, die Aufträge, Rollen und $\mathrm{Zu}$ ständigkeiten für Aktivitäten der Kinder- und Jugendarbeit an der Schule einschließlich Standards klar beschreiben.

\section{Auswirkungen bei der Hilfe zur Erziehung}

Die freien Träger von Hilfen zur Erziehung sehen die Ganztagsschulen als einen relevanten neuen Arbeitsbereich und befassen sich bewusst mit Fragen der Neuausrichtung und Integration ihrer Angebote an Ganztagsschulen.

In Zukunft werden ihrer Einschätzung nach flexible Hilfen, soziale Gruppenarbeit und Leistungen der Erziehungsberatung, die Beratung in oder bezogen auf Schule anbieten deutlich zunehmen. Eine abnehmende Tendenz wird bei Tagesgruppen gesehen. Im Un- terschied zur Jugendarbeit sind die befragten Träger der Auffassung, ihre spezifische Qualität auch bei Angeboten in Ganztagsschulen beibehalten zu können.

Die freien Träger von Hilfen zur Erziehung sind bislang nur in geringem Umfang in ganztagsschulbezogene Netzwerke eingebunden und bilden selbst keine Kooperationsverbünde für abgestimmte Aktivitäten an Ganztagsschulen. Auch von einer Begleitung durch den Allgemeinen Sozialen Dienst des Jugendamts bei der Ausrichtung ihrer Angebote auf Ganztagsschulen berichten die Träger von Hilfen zur Erziehung eher nicht.

Die Angebote des Leistungsfeldes Hilfe zur Erziehung werden sich zukünftig durch den Ausbau der Ganztagsschulen verändern müssen. Es bedarf einer gezielten Steuerung, um Angebote der erzieherischen Förderung und Ganztagsschulen zu verbinden und Unterstützungsbedarfen von Kindern, Jugendlichen und $\mathrm{Fa}$ milien entsprechen zu können. Die in Ganztagsschule integrierten Angebote haben dabei häufig einen niederschwelligen Charakter und erleichtern Eltern und Familien den Zugang.

Insbesondere an den Standorten, an denen bestimmte teilstationäre Leistungsformen wie die Tagesgruppe keinen Bestand mehr haben und beispielsweise durch Integrative Soziale Gruppenarbeit an Ganztagsschulen ersetzt werden, stellt sich die Frage, wie diese Lücke geschlossen werden kann. Denn der Bedarf an Hilfen zur Erziehung entsteht nicht nur im schulischen Kontext und nicht jedem Bedarf an Hilfe zur Erziehung kann im Rahmen einer Ganztagsschule angemessen entsprochen werden.

In diesem Zusammenhang gilt es künftig auch zu klären, wie Kindern und Jugendlichen in vollstationären Formen

\section{Forschung und Fortbildung}

Der Kommunalverband für Jugend und Soziales Baden-Württemberg ist ein Kompetenz- und Dienstleistungszentrum für die 44 Stadt- und Landkreise Baden-Württembergs. Er nimmt in seinem Dezernat Jugend - Landesjugendamt die Aufgaben des überörtlichen Trägers der Kinder- und Jugendhilfe wahr. Der Kommunalverband für Jugend und Soziales Baden-Württemberg entwickelt und begleitet Forschungsvorhaben zu praxisrelevanten Feldern der
Sozialen Arbeit. Deren Ergebnisse sind im Internet veröffentlicht. Dort wird auch ein breites Spektrum an Fortbildungsveranstaltungen angeboten. Zum Thema Ganztagsschule gibt es beispielsweise gemeinsame Angebote des Kommunalverbands für Jugend und Soziales Baden-Württemberg mit der Serviceagentur "Ganztägig lernen« Baden-Württemberg.

www.kvjs.de/Forschung www.kvjs.de/Fortbildung 


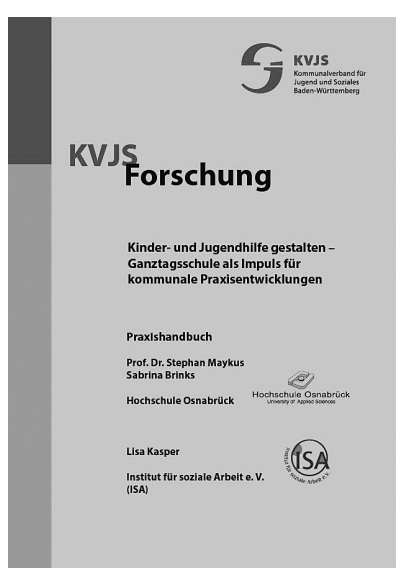

Aus dem Forschungsvorhaben "Auswirkungen des Ausbaus der Ganztagsschulen auf die Strukturen und Arbeitsweisen der Kinder- und Jugendhilfe in Baden-Württemberg" gingen vier Materialbände mit umfangreichen Ergebnissen der quantitativen und qualitativen Erhebungen sowie ein Praxishandbuch hervor, die alle im Internet frei zugänglich zur Verfügung stehen.

www.kvjs.de/forschung/

ganztagesschule.html

der Heimerziehung, die bislang nicht die Sonderschule am Heim, sondern die allgemeine Schule am Ort besucht haben, weiterhin der Besuch dieser allgemeinen Schule ermöglicht werden kann, wenn diese Ganztagsschule wird.

\section{Auswirkungen bei der Kindertagesbetreuung}

Die Ganztagsschule bietet der Kindertagesbetreuung neue Chancen durch integrierte Bildungs- und Betreuungsangebote. Die aufeinander abgestimmten Betreuungskonzepte zwischen Schule und sozialräumlichen Betreuungsformen können den Familien ein lückenloses Betreuungsangebot gewährleisten. Durch die Betreuung aus einer Hand können zudem der Blick für mögliche Unterstützungsbedarfe erweitert und Fördermöglichkeiten für Kinder optimiert werden. Nicht zuletzt kann die Verlagerung der Schulkindbetreuung in die Ganztagsschule auch mögliche Kapazitäten für den Ausbau der Kleinkindbetreuung freisetzen.

Bislang scheint die Gestaltung der zukünftigen Schulkindbetreuung jedoch noch einen geringen Stellenwert in den Jugendämtern einzunehmen. Der Aus- bau der Kleinkindbetreuung dominiert derzeit die öffentlichen und fachinternen Diskussionen. Bezogen auf die Schulkindbetreuung scheint den Fachberatungen der Kindertagesbetreuung der Stellenwert des Ganztagsschulausbaus und vor allem ihre Rolle innerhalb dieser Neustrukturierungen vielerorts noch nicht bewusst zu sein. Dabei braucht es zur Gestaltung bedarfsgerechter Schulkindbetreuung in der Ganztagsschule eine enge Abstimmung zwischen Kindertagesbetreuung und Schule. Hierzu müssen die Wünsche der Eltern erfasst und auf möglichst kleinräumlicher Ebene gestaltet werden.

Mit dem Wechsel der Schulkindbetreuung von Horten in die Ganztagsschulen entfällt die Einhaltung der Mindesterfordernisse für die Betriebserlaubnis als Einrichtung der Jugendhilfe. Dennoch muss die Betreuungsqualität in der Schule gewährleistet bleiben. Dies kann nur gelingen, wenn sich die Kindertagesbetreuung und die Schule gemeinsam verlässliche Standards und Rahmenbedingungen erarbeiten und dafür die Erfahrungen der Horte nutzen. Und wenn die Betreuung an den Ganztagsschulen durch Träger und Fachkräfte erfolgt, die sich fachlich als Teil der Kinder- und Jugendhilfe sehen.

\section{Erwartungen an das Land}

Entscheidende Rahmenbedingungen für das Zusammenwirken von Jugendhilfe und Ganztagsschule muss das Land schaffen. Die überfällige gesetzliche Regelung der Ganztagsschule muss auch förderliche Rahmenbedingungen für das Zusammenwirken mit der Jugendhilfe beinhalten. Gleichermaßen gilt dies für die angekündigte gesetzliche Regelung der regionalen Schulentwicklung.

\section{Anmerkungen}

(1) »Der Wechsel beginnt« Koalitionsvertrag zwischen Bündnis90/DIE GRÜNEN und der SPD BadenWürttemberg, Baden-Württemberg 2011-2016, Seite 7.

(2) Wir bitten um Verständnis, dass aus Gründen der Lesbarkeit auf eine durchgängige Nennung der weiblichen und männlichen Bezeichnungen verzichtet wird. Selbstverständlich beziehen sich die Texte in gleicher Weise auf Frauen und Männer.

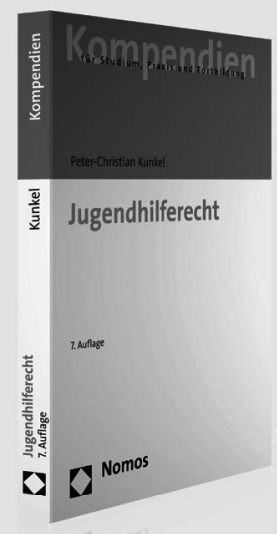

Jugendhilferecht

Systematische Darstellung für Studium und Praxis

Von Prof. Peter-Christian Kunkel

7. völlig neu bearbeitete

Auflage 2013, $490 \mathrm{~S}$.,

brosch., 25,-€

ISBN 978-3-8329-7778-8

Das bereits in 7. Auflage erscheinende Kompendium von Kunkel ist ein fester Bestandteil für Ausbildung und Praxis der Jugendhilfe. Zahlreiche Schaubilder, vertiefende Beispielsfälle, Übersichten über Rechtsquellen und Rechtsprechung, Hinweise auf Arbeitshilfen, Verwaltungsvorschriften und Mustersatzungen ermöglichen einen didaktisch ausgewogenen wie an der Verwaltungspraxis ausgerichteten Zugang zum gesamten Recht der Kinder- und Jugendhilfe einschließlich des Datenschutzes.

Die aktuelle Auflage berücksichtigt insbesondere die neuen Regelungen des Bundeskinderschutzgesetzes. Dessen Auswirkungen für die tägliche Verwaltungspraxis werden anhand konkreter Anwendungsfälle deutlich gemacht.

www.nomos-shop.de/19715

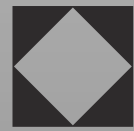

Nomos 